\section{Neuausrichtungen}

Die DDG ist seit Jahrzehnten zumindest organisatorisch eine Baustelle, auch auf der diesjährigen Jahrestagung in Stuttgart referierte der DDG-Präsident ausführlich über eine Neuorientierung. Wegen interner Streitigkeiten wurde ein neuer Patientenverband gegründet, der künftig unter dem Label „Deutsche Diabetes-Hilfe - Menschen mit Diabetes e.V." (DDH-M) unter dem Dach von diabetesDE auf Zuspruch hofft.

Notwendig wurde dieser Beschluss, weil drei Landesverbände des Deutschen Diabetiker Bundes (DDB), Nordrhein-Westfalen, Rheinland-Pfalz und Bremen, ihren Austritt aus dem DDB erklärt haben. „Mit dieser Neugründung möchten wir den drei Landesverbänden eine neue Heimat bieten, aber auch den Einzelmitgliedern und den freien Selbsthilfegruppen in diabetesDE gerecht werden. Die Gründung einer bundesweiten eigenständigen Organisation für Menschen mit Diabetes ist der einzig sinnvolle Weg, diabetesbetroffenen Menschen innerhalb von diabetesDE auch strukturell ein Gleichgewicht zu den Ärzten und Beratern zu geben", erklärte Prof. Dr. Thomas Danne, Vorstands- vorsitzender von diabetesDE - Deutsche Diabetes-Hilfe.

Nationaler Diabetes-Plan: Work in progress - forever ?

Und dann wird wieder einmal an einem nationalen Diabetes-Plan gearbeitet. Nicht das erste Mal, diesmal aber auf Druck der UNO, die alle Länder aufforderte, sich mit einem nationalen Aktionsplan auf die Zunahme des Diabetes vorzubereiten bzw. einzurichten. Der Interessenausgleich zwischen den diversen Fachgesellschaften sowie Diabetes-Bünden und -Verbänden dauert schon Jahre, inzwischen sind andere europäische Länder dem UNO-Aufruf längst gefolgt.

\section{Register in Hülle und Fülle}

Auch über nationale Register wird wieder diskutiert. Diesmal steht im Mittelpunkt und mit eigenem Stand in der KongressAusstellung DIVE (Diabetes VersorgungsEvaluation - Eine Nationale Initiative zur Qualitätssicherung in der diabetologischen Versorgung). Die Datenerhebung erfolgte im April in 178 diabetologischen Schwerpunktpraxen unter Ver-

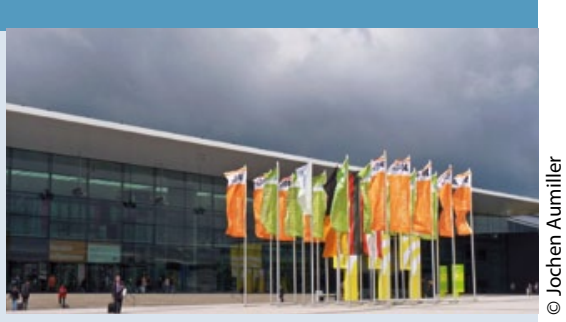

Wann klärt sich der Himmel über der DDG?

wendung einer einheitlichen Praxissoftware (Diamax); Sponsor: Sanofi-Aventis. Letztlich soll DIVE in der Etablierung eines Nationalen Diabetes Registers münden, hofft Prof. Thomas Danne. Man kann ihm nur viel Glück wünschen, denn dies ist nicht die erste Registeriniative. Erinnert sei an HYDRA, DUTY, CoDim, DIG, SweetHeart, DiaREGIS, CODE-2, ROSSO. Außerdem werden derzeit noch andere Register geplant, z. B. DIALOGUE, angekündigt als „weltweit erstes Register zu den Ergebnissen einer kombinierten antidiabetischen und antihypertensiven Therapie"; Sponsor: Novartis.

Man darf in diesem Zusammenhang vielleicht doch einmal darum bitten, von kompetenter und unabhängiger Stelle zu erfahren, was all diese Register bislang gebracht haben und wie viele Ressourcen dafür verbraucht wurden.

Jochen Aumiller I

\title{
Antidiabetische Therapie
}

\section{Sulfonylharnstoffe in Erklärungsnöten}

\author{
Über die optimal antidiabetische The- \\ rapie wird trefflich gestritten, auch in \\ Stuttgart widmeten sich wieder zahl- \\ reiche Sitzungen den medikamentösen \\ Optimierungschancen. Immer mehr in \\ Bedrängnis scheint die Gruppe der Sul- \\ fonylharnstoffe zu kommen, nicht zu- \\ letzt auch aus ökonomischen Gründen, \\ sie kosten deutlich weniger als die neu- \\ en Inkretin-Derivate. Prof. Thomas \\ Forst, Mainz, gibt allerdings zu beden- \\ ken, dass erhöhe Mortalitätsraten in \\ populationsbasierten Studien Anlass \\ zur Sorge geben.
}

IL Bereits vor 30 Jahren wurde die Sicherheit von Sulfonylharnstoffen (Sus) kontrovers diskutiert, angefacht von der UGDP-Studie. Ihre Ergebnisse ließen kardiovaskuläre Probleme mit Tolbutamid vermuten, ein erstes Signal. In Populationsstudien leuchtete dieses Signal immer wieder auf und in einer kürzlich publizierten Metaanalyse loderte es geradezu. 17 populationsbasierte Studien mit mehr als 500000 Typ-2-Diabetikern wurden gepoolt, es ergab sich Forst zufolge eine um 92\% erhöhte Gesamtmortalität in der Gruppe der mit Sus behandelten Patienten im Vergleich zu den Gruppen, die andere Substanzen erhielten. Forst: „Vergleicht man Sus-ba- sierte Monotherapien mit allen anderen Formen der Monotherapien ergibt sich sogar eine um 148\% erhöhte Gesamtmortalität." Weshalb es zu dieser Exzessmortalität kommt, ist letztlich nicht geklärt. Angeschuldigt werden mehrere Pathomechanismen. Zunächst die Gewichtszunahme unter den Sus. Die Metaanalyse zeigte sogar eine deutlich gesteigerte Sterblichkeit bei einem BMI von 30 $\mathrm{kg} / \mathrm{m}^{2}$ aufwärts.

Weiterhin im Vordergrund der Erklärungsversuche stehen gehäufte Hypoglykämien, in denen man im Gegensatz zu vor 20 Jahren heute einen ernsten Risikofaktor für kardiovaskuläre Erkrankungen sieht. 